\title{
La incidencia de la inteligencia de negocios en la gestión gerencial de las empresas del mueble y la madera de Bogotá D.C.
}

\section{The incidence of business intelligence in the management of furniture and wood companies in Bogotá D.C}

\author{
Andrés Rocha Alfonso ${ }^{1}$
}

DOI: 10.29151/hojasyhablas.n18a9

\section{Resumen}

En Colombia, el sector del mueble y la madera ha mantenido una participación en el Producto Interno Bruto (PIB) del 0.67\% del año 2012 al 2017 (Encuesta Anual de Manufactura, 2016), manifestando un estancamiento en un mercado dinámico. El presente artículo muestra los factores que afectan el desarrollo del sector y su relación con la inteligencia de negocios en la dirección de las empresas. Esta investigación se basó en un enfoque mixto, desde la percepción de representantes del sector, indagando por los factores que inciden en la falta de competitividad y el análisis frente al uso de herramientas tecnológicas en la gestión de los empresarios. Los cuestionarios y entrevistas se realizaron en la XIV Feria Tecnológica e Industrial del Mueble y la Madera en Centro Internacional de Negocios y Exposiciones de Bogotá (Corferias) en el año 2018, con el fin de identificar las variables de competitividad de las empresas, conocer la gestión de la inteligencia de negocios en la dirección de las micro y pequeñas empresas, y dar cuenta de las entidades que promueven el desarrollo de las empresas del mueble y la madera por medio de la capacitación y la tecnificación.

Palabras claves: Industria del mueble; sistemas de información; inteligencia de negocios; toma de decisiones; competitividad.

\begin{abstract}
In Colombia, the furniture and wood sector has maintained a participation in the Gross Domestic Product (GDP) of $0.67 \%$ from 2012 to 2017 (Annual Manufacturing Survey, 2016), the growth of the national industry has stagnated in a market dynamic, therefore, this article shows the factors that affect the development of the sector and its relationship with business intelligence in the management of companies. The study was based on a mixed approach, from the perception of the representatives of the sector, investigating the factors that affect the lack of competitiveness and the analysis of use of technological tools in the management of entrepreneurs. The questionnaires and interviews were conducted at the XIV Feria Tecnológica e Industrial del Mueble y la Madera in the International Business and Exhibition Center of Bogotá (Corferias in Spanish) in 2018, in order to identify the variables of competitiveness of companies, to know the management of business intelligence in the direction of micro and small businesses, and to identify the entities that promote the development of furniture and wood companies.
\end{abstract}

Keywords: Furniture industry; information systems; business intelligence; decision making; competitiveness.

\footnotetext{
${ }^{1}$ Administrador de Empresas, Magíster en Administración de Empresas con especialidad en Dirección de Proyectos. Candidato a Doctor en Administración con énfasis en Investigación. Integrante del Grupo GENENE. E-mail: arochaa@unimonserrate.edu.co. ORCID: https://orcid.org/0000-0001-5926-3567
} 


\section{Introducción}

La industria del mueble en Bogotá es reconocida por la especialización en su producción, lo anterior, ha generado un posicionamiento que ha beneficiado a aquellas personas jurídicas que tienen actividades económicas similares o complementarias y que se han unido para establecer estrategias conjuntas, donde la demanda puede encontrar variedad en diseño, calidad y precios. Las pequeñas y medianas empresas, dedicadas a esta actividad, tienen que enfrentar diariamente situaciones que comprometen su subsistencia por falta de información que permita responder a las exigencias del mercado, sumado a la volatilidad de las variables externas e internas que inciden en del desarrollo de la actividad económica de las empresas. A partir de esta situación, se plantea la pregunta de investigación ¿Cómo las empresas del sector del mueble y la madera de Bogotá, utilizan la inteligencia de negocios para la toma de decisiones gerenciales?

Para responder a esta pregunta, el artículo se estructura en tres partes. En la primera sección, se identifica la situación actual del sector; en la segunda, se establece la definición y percepción de la inteligencia de negocios que tienen representantes y empresarios de la industria de la madera, quienes asistieron al evento de la industria. Y por último, se caracterizan las entidades que promueven el desarrollo del sector.

El estudio se basa en una muestra a conveniencia, debido a que algunas empresas del mueble y la madera desarrollan su actividad desde la informalidad, lo que impide conocer un universo y seleccionar una muestra representativa, por lo anterior, se escogió la XIV Feria Tecnológica e Industrial del Mueble y la Madera en Centro Internacional de Negocios y Exposiciones de Bogotá (Corferias) en el año 2018, donde los representantes del sector y de las empresas asistieron a conocer las nuevas tendencias de la industria. Hernández, Fernández y Baptista (2014 citando a
Battaglia, 2008a) afirman que estas muestras están conformadas por los casos disponibles a los cuales tenernos acceso. La información se obtuvo gracias al apoyo de los representantes de la Corporación Autónoma Regional (CAR), Ministerio de Comercio Industria y Turismo, y empresarios.

\section{La inteligencia de negocios y la gestión gerencial}

La Business Intelligence (BI) tiene como objetivo el almacenamiento y manejo de datos (minería de datos) que se generan en todos los procesos internos y externos que tiene una organización. Lo anterior no es nuevo, ya que en una gestión gerencial tradicional la información siempre ha sido importante para la toma de decisiones, sobre todo aquella que tiene que ver con las áreas de mercadeo, finanzas o producción. Pero desde una perspectiva más dinámica, la BI establece patrones de comportamiento de los procesos internos de la empresa, de la economía de un país, del mercado cautivo o de la competencia, todo lo anterior de manera inmediata y veraz. La eficiencia de la información, conlleva a que la planeación estratégica sea capaz de responder ante los cambios de manera oportuna y eficaz.

A partir de la gestión del conocimiento, surge el concepto de inteligencia de negocios (Business Intelligence, inteligencia empresarial o inteligencia de negocios); se llama así al conjunto de estrategias, acciones y herramientas enfocadas a la administración y creación de conocimiento mediante el análisis de datos existentes en una organización o empresa (Ahumada y Perusquia, 2016).

Gracias a la BI, la elaboración de un plan estratégico adquiere mayor complejidad, pues se concibe desde diferentes puntos de vista por medio de una planeación participativa, en donde los actores involucrados (stakeholders) establecen el direccionamiento adecuado de la organización por medio de los datos obtenidos en el desarrollo de sus actividades diarias. Una operación que puede explicar esta dinámica es la compra de un 
producto o servicio, todas las áreas o unidades estratégicas de negocios de una empresa que hacen parte de una operación comercial. Cada registro alimenta de manera inmediata el movimiento de los inventarios, establece las requisiciones al departamento de compras, genera solicitudes a los proveedores, alimenta los estados financieros, caracteriza a los clientes y activa las áreas que participan de este proceso en un tiempo simultáneo a aquel en que el comprador está adquiriendo su producto o servicio.

En la actualidad, la BI se basa en la industria 4.0 que integra la sociedad con la tecnología, esta postura innovadora está generando cambios en todos los modelo de gestión organizacional, al transformar la perspectiva de la gestión en todos los eslabones de una cadena de suministros, ya sea un bien o un servicio, donde se involucran: big data y análisis de datos, computación en la nube, ciberseguridad, internet de las cosas, integración de procesos, robótica avanzada e inteligencia artificial, sistemas para la integración vertical y horizontal, hiperconectividad, fabricación digital (3D), sistemas de ejecución de fabricación software y realidad virtual y aumentada. Esta tendencia tiene una postura constructiva, pues genera un escenario donde la gestión de conocimiento es la principal herramienta para generar oportunidades. El término industria 4.0 se refiere a un nuevo modelo de organización y de control de la cadena de valor a través del ciclo de vida del producto y a lo largo de los sistemas de fabricación apoyado y hecho posible por las tecnologías de la información (Román, 2019).

La planeación estratégica, o la inteligencia estratégica en las organizaciones, se logra gracias a información veraz e idónea que se deriva de los Sistemas de Información Gerencial (SIG), estos se estructuran desde el nivel operativo, ejecutivo y gerencial. La dirección organizacional utiliza esta información para tomar decisiones con base en un argumento válido derivado de investigaciones, informes sectoriales, comparativos con la compe- tencia y todos los datos que se generan en el desarrollo diario de la empresa y que son almacenados para depurar, analizar y utilizar en un plan de negocios. Los SIG son diseñados para controlar el cumplimiento de los objetivos y generar pronósticos que permitan crear escenarios estratégicos e identificar las oportunidades y los riesgos que pueden afectar el direccionamiento de la empresa. Aguirre (2015) ha definido la inteligencia estratégica como un conocimiento específico, codificado, secreto y oculto que manejan algunas entidades militares y gubernamentales, concebido como un producto integrado a la seguridad nacional y desarrollo de un país (Garden, 2003; Nelson y Rose, 2012).

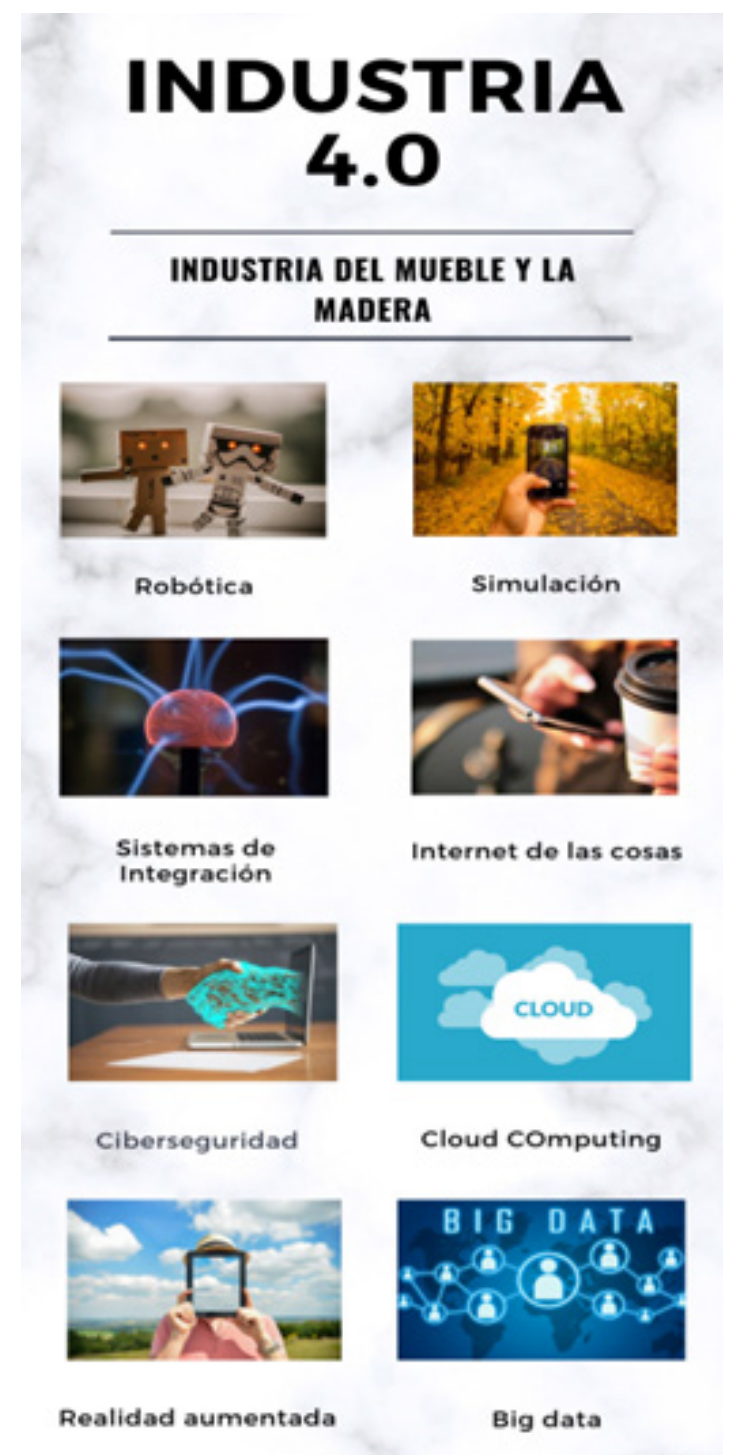


Con base en lo anterior, la minería de datos identifica las actividades que generan valor a la organización y también establece las oportunidades para optimizar la gestión en las operaciones de cada unidad estratégica de negocios, con el fin de establecer una la cultura de mejoramiento continuo. Según Chirinos et. al (2010), kaizen o mejoramiento continuo implica a todas las personas, tanto gerentes como trabajadores. Se basa en el seguimiento del servicio, esta información caracteriza aquellos factores que causan demoras o reprocesos en el interior de la organización y permite conocer las inconformidades o sugerencias que tiene el mercado y que afecta una frecuencia de compra, si no son atendidas de manera oportuna.

Un medio para conocer las perspectivas de los clientes internos y externos son las Peticiones, Quejas y Reclamos (PQRs) que se articulan con el plan de negocios para crear acciones correctivas y preventivas que mitiguen el impacto de las actividades que no tiene una gestión diligente. La interacción con el cliente y atender a sus observaciones, se convierten en una estrategia de fidelidad que integra a todos los participantes en una dirección participativa de la empresa.

La BI depende de las habilidades del personal, y con base en lo anterior, las competencias de desempeño deben ser perfeccionadas por la organización de acuerdo a los requerimientos del mercado. Ahumada y Perusquia (2016) establecen que la gestión del conocimiento es donde se encierra las variables de los sistemas de información, se estructura modelos para la toma de decisiones con el fin de minimizar tiempos de respuesta en cada uno de los procesos y optimizar los recursos enfocados a la meta de la creación de valor.

En el proceso de toma de decisiones, la tecnología permite tener información pertinente y veraz, en consecuencia, las empresas y sobre todo aquellas que tienen actividades de comercio exterior, deben contemplar este factor en sus partidas presupuestales.
Las Tecnologías, Información y Comunicación (TIC'S) ayudan a las negociaciones internacionales a través de las diferentes etapas tales son: preparación, dialogo y cierre; las empresas usan estas grandes tecnologías para comunicarse y gestionar grandes negocios haciéndolo más rápidos y eficientes. Todas las organizaciones tienen la posibilidad de aprovechar, utilizar e implementar las tics en cuando a las necesidades que se tengan dentro de estas. (Cano, 2015, p. 337)

La diligente utilización de la información conlleva a organizaciones innovadoras. Dicho término se basa en el conocimiento de lo partícipes de una organización, en consecuencia, las empresas estructuran un plan de negocios direccionado a la competitividad. En la industria del mueble y la madera se ha tratado de fortalecer los procesos de producción, mercadeo, organización y dirección con políticas que buscan el fortalecimiento de las instituciones que hacen parte de este sector. Mendoza y Valenzuela (2014) afirma que "un problema generalizado entre las micro, pequeñas y medianas empresas (Pymes) es su escasa capacidad para ofrecer productos o servicios diferenciados e innovadores debido, en parte, al bajo grado de complejidad tecnológica que poseen" (p. 273).

La formación que reciben los directivos influye favorablemente en la innovación de productos, servicios y sistemas productivos sobre la introducción de nuevas tecnologías que realiza la empresa" (Barba, 2014). Para finalizar, se enfatiza en el hecho de que los recursos humanos son un activo muy valioso para que las empresas consigan innovar y obtener una ventaja competitiva sostenible.

Bernal, Frost y Sierra (2015) afirman que el conocimiento empírico de una organización depende mucho de las características de su líder, ellos requieren de sistemas de información adecuados para organizar dirigir y planear estrategias en cada una de las actividades, en la investigación el autor presenta cómo las pequeñas empresas 
toman decisiones basadas en la experiencia y no implementan las herramientas necesarias para la obtención de información, factor que incide en que las empresas no sean competitivas y en un futuro puedan desaparecer del mercado por la obsolescencia en sus procesos. La afirmación anterior, establece una gestión basada en el conocimiento empírico más no el metodológico, por eso es necesario establecer en los antecedentes las herramientas para la obtención y análisis de la información que direccionan la toma de decisiones gerenciales. Este estudio se realizó a 2.249 personas en 83 organizaciones, los resultados muestran como la Tecnología Información y Comunicación ocupa un lugar secundario en dinámica empresarial y prima el aprovechamiento del talento humano como factor clave para el desarrollo de las actividades.

Leyva, Pérez, Febles y Gulín (2013), resaltan la BI como una herramienta poco utilizada, pero de gran aporte en los procesos de una organización, la cual brinda un soporte metodológico al análisis de varios escenarios, dando así una visión más clara, a la alta gerencia, de la toma de decisiones. La herramienta tiene alta flexibilidad y adaptabilidad a cualquier organización, es una estrategia efectiva basada en las nuevas tecnologías que pueden llegar a ser bastantes útiles en una organización para ser cada vez más eficiente. En la investigación The Internet of Things: Building a knowledge management system for open innovation, Santoro (2017) hace un hardware que gestiona el conocimiento a través de la digitalización donde las empresas puedan consultar y generar un aporte, con el fin de ser eficientes y tomar lineamientos basados en los conocimientos.

La BI se estructura en SIG, que garantizan la calidad y veracidad de cada uno de los datos a utilizar, Abrego, Sánchez y Medina (2016) expone la importancia de los SIG para cada uno de los procesos de la organización y su incidencia en el clima organizacional. La gestión del conocimiento depende de los SIG en la operación de las organizaciones, la calidad y veracidad es indispensable en cada uno de los datos a utilizar, ya que la organización se vuelve mucho más eficaz y establece un proceso de reingeniería donde se pueden optimizar los procesos.

En la formación de líderes, la BI recomienda planes de capacitación pertinentes, con el fin de mejorar las habilidades del equipo de trabajo para una dirección empresarial en condiciones complejas, donde se necesita una gestión administrativa proactiva basada en argumentos idóneos. Tena-Chollet, Tixier, Dandrieux, y Slangen (2016) resaltan la importancia de la toma de decisiones en situaciones de crisis para minimizar las consecuencias potenciales de una serie de eventos que necesitan de las habilidades directivas para encaminar la organización a un escenario de oportunidades.

Las empresas entienden la importancia de desarrollar las competencias de los trabajadores y con base en lo anterior crean diferentes estrategias de formación con instituciones de educación formal o informal, cursos que están direccionados a necesidades específicas de las organizaciones. Para optimizar el tiempo y la calidad de vida del trabajador, el aprendizaje se promueve con la modalidad e-learning, que hace parte de la industria 4.0. Frente a esto, Chang (2016) resalta la importancia de este tipo de educación al emplear enfoques basados en la tecnología desde cualquier lugar y en cualquier momento. Como se ve, la educación virtual es un mecanismo para adquirir nuevos conocimientos y para que las empresas puedan ser más competitivas en el mercado.

La BI utiliza simuladores gerenciales para mejorar los resultados en el proceso de formación, Luigi (2015) menciona que a través de plataformas se pueden reducir tiempos y movimientos en un modelo de producción. El simulador ayuda en todos los aspectos a entrenar a los trabajadores y a que tengan herramientas de cómo actuar en un caso de adversidad que se les presente en su ámbito de trabajo. En el BI la simulación gerencial es 
una herramienta para crear escenarios empresariales, identificar y jerarquizar las variables externas e internas que más inciden en el posicionamiento y en la rentabilidad de una organización, al establecer estos factores se ponderan según el nivel de riesgo y se hace la selección en beneficio de los objetivos empresariales. Los simuladores gerenciales o juegos gerenciales, reflejan los indicadores que diagnostican la situación de una organización, estos son: ingresos, beneficio bruto, rentabilidad sobre el patrimonio, ratio de endeudamiento, unidades vendidas, participación en el mercado, tasa de ocupación de la fábrica, valor de marca, nivel de diseño, eficiencia en la productividad y capacidades organizacionales. No significa que los anteriores indicadores sean los únicos que se pueden utilizar, pero si son los más importantes para conocer el situación actual de la empresa y tomar decisiones correctivas o preventivas.

Vidal y Domenge (2017), explica cómo un simulador de vuelo gerencial surge de la necesidad de conocer los resultados de una estrategia y no esperar a una crisis financiera.

Los simuladores pueden ser utilizados como parte del proceso de planeación estratégica y de aprendizaje de una empresa y ayudan a los estrategas a distinguir la estructura de retroalimentación del sistema (empresa) y cómo modificar dicho sistema existente para afectar su comportamiento, buscando los objetivos previamente planteados. La planeación puede ser concebida como un medio de aprendizaje y el simulador de vuelo empresarial como vehículo que da soporte y un mejor entendimiento del sistema de negocios en cuestión (Bianchi et al., 1998) para la toma de decisiones más informada. (p. 564)

Otro escenario es la simulación en la dirección empresarial. Orta, Hurtado y Gawn (2014) explican que el modelo de simulación se usa ampliamente para apoyar los procesos en la Gestión de Servicios de Tecnologías de la Información o IT Service Management (ITSM), se centra en la aplicación de modelos de simulación para apoyar toma de decisiones en el ámbito de ITSM, los enfoques de simulación se están utilizando para abordar muchos problemas en el contexto de varios procesos utilizando el Big Data (BD), que amplía la adquisición, almacenamiento, minería, limpieza, análisis y modelado e interpretando datos. (Sivarajah, Mustafa, Irani y Weerakkod, 2016).

Allal-Chérif y Bidan (2016) muestran que los aportes de simuladores se encuentran en el reclutamiento de personal de L'Oreal Reveal, IBM, INNOV8, CityOne y Thales Moonshield, se convierten en juegos serios usados dentro de empresas para reclutar y entrenar empleados. Como se puede evidenciar, esta herramienta puede ser de gran ayuda para las organizaciones ya que brindan una forma distinta e innovadora de capacitar a sus empleados, y a su vez es una plataforma que ayuda a elegir e integrar nuevos miembros dentro de las organizaciones.

\section{La Industria del mueble y la madera}

La industria del mueble y la madera en Colombia es un eje fundamental para el desarrollo económico del país, ya que dinamiza otras organizaciones empresariales que tienen relación con esta actividad. Esta industria alberga instituciones con un amplio portafolio de productos que han beneficiado al mercado nacional durante varios años por su innovación y calidad, lo anterior es el resultado de una fabricación artesanal y una habilidad desarrollada por varias generaciones.

La Encuesta Anual Manufacturera, DANE (2017) registra 313 establecimientos de fabricación de mueble en Colombia, con 17.365 personas ocupadas donde 10.037 están remuneradas de manera permanente y 3.598 de manera temporal. Bogotá representa el $40.71 \%$ de estas estadísticas. Según la Revista Gerente (2018), la industria del mueble ha tenido un crecimiento en los últimos años y tiene buenas perspectivas. Según datos del Euromonitor (2018), el valor de la producción de muebles en Colombia creció anualmente $3,1 \%$ en términos reales del 2011 al 2016, en promedio. 
En el año 2017, el sector de la madera ha incrementado las importaciones en $1.4 \%$ (Revista Gerente, 2018), lo que evidencia que la producción nacional no tiene la capacidad para satisfacer la demanda interna, uno de los factores que inciden en la situación anterior, es la falta de inversión en maquinaria, tecnología e investigación. Teniendo en cuenta el comportamiento de la producción, es complejo pensar en ingresar a mercados externos donde los requerimientos en cantidad son mayores a la capacidad instalada de la industria nacional.

Según Díaz (2018), factores como el aumento de los impuestos o la incertidumbre en la situación laboral, ha afectado la compra de muebles en el país, afirmando que "las empresas han tenido que reinventarse y, en el caso de los muebles, ofrecer diseños exclusivos y a precios justos, pero en línea con las tendencias globales" (párr. 5). La industria de los muebles se caracteriza por contar con organizaciones que están catalogadas dentro de las microempresas y pequeñas empresas. El direccionamiento administrativo de estas organizaciones se encuentra bajo la responsabilidad de personas que han adquirido su conocimiento de una forma empírica, y a través del tiempo han desarrollado el arte de la negociación y la habilidad de desarrollar productos de forma artesanal que satisfacen las expectativas del mercado. La falta de competitividad en el sector, se debe a la ausencia de factores como falta de estándares internacionales de calidad, diseños tradicionales, tendencias de gestión empresarial tradicionales y seguir en el mismo mercado. Lo anterior, se suma a una cultura individualista donde los empresarios no hacen alianzas estratégicas o se vinculan a organizaciones de fomento y apoyo.

\section{Metodología}

Para conocer la percepción de los empresarios del sector de muebles en el uso de la inteligencia de negocios en la gestión empresarial, se realizó el estudio en la XIV Feria del Mueble y la
Madera celebrada en el año 2018 en Corferias-Bogotá. En este evento participaron 192 expositores y varios ponentes de entidades públicas y privadas, que promueven el desarrollo en esta industria. En el estudio se realizaron tres entrevistas en profundidad a funcionarios del Ministerio de Comercio, Industria y Turismo; y la Corporación Autónoma Regional, quienes expusieron sus opiniones respecto a los factores que obstaculizan la competitividad en la micro y pequeña empresa. También se contó con la percepción de diez empresas que son las más representativas del sector y son catalogadas como grandes empresas y diez mico y pequeñas empresas quienes relacionaron el uso de inteligencia de negocios en sus organizaciones.

El anterior muestreo se hizo a conveniencia no probabilística, con el fin de conocer la opinión de personas con las que se podían tener acceso y por su trayectoria son representativos en toda la población. En la entrevistas con funcionarios públicos se utilizó una entrevista semiestructura para que pudieran manifestar su opinión de forma espontánea sin ningún tipo de sesgo, y en las encuestas con empresarios se utilizaron once variables de inteligencia de negocios como: big data y análisis de datos, computación en la nube, ciberseguridad, internet de las cosas, integración de procesos, robótica avanzada e inteligencia artificial, sistemas para la integración vertical y horizontal, hiperconectividad, fabricación digital (3D), sistemas de ejecución de fabricación software y realidad virtual y aumentada. Estas variables fueron medidas con una escala tipo liker con cinco escenarios: muy importante, importante, moderadamente importante, de poca importancia y sin importancia. Las encuestas a grades empresarios y pequeños empresario permitieron dimensionar la diferencia de gestión administrativa con base en el tamaño de la empresa.

\section{Resultados y discusión}

En 2018, durante la XIV Feria se reunieron 192 expositores y 13.000 visitantes interesados en 
conocer las nuevas tendencias que tiene el sector, y en este evento se desarrollaron entrevistas con especialistas para conocer la percepción que tienen de la industria 4.0 en las empresas que fabrican y comercializan muebles y maderas. Estos expertos son funcionarios del Ministerio de Comercio, Industria y Turismo y la Corporación Autónoma Regional - Bogotá, quienes promueven el desarrollo de la industria maderera por medio de asesorías y acompañamiento. En las entrevistas se establecieron las siguientes variables como aquellas que más inciden en la competitividad de las empresas:

\section{Figura No. 1. Principales factores que inciden en la competitividad de las empresas.}

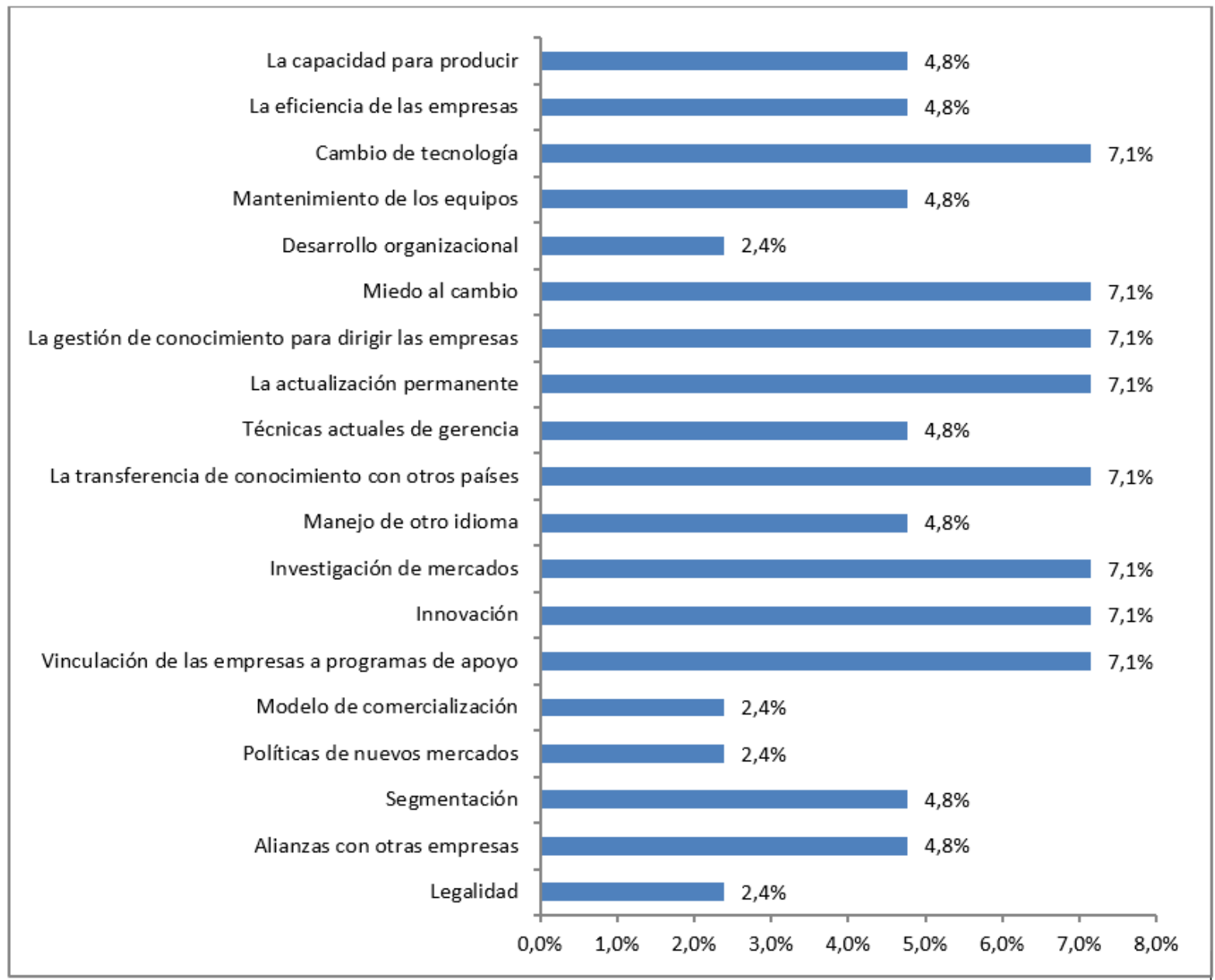

Fuente: Elaboración propia.

Las personas entrevistadas destacaron algunos aspectos, con miras a que las empresas de la industria de la madera sean competitivas. Cabe resaltar que, este instrumento no tenía ningún condicionamiento ni direccionamiento, y que sus repuestas se dieron de manera espontánea. En el análisis se identificaron las respuestas con mayores coincidencias entre las tres personas. Al relacionar las respuestas con las variables de la inteligencia de negocios con base en la industria 4.0, las respuestas se sintetizaron de la siguiente manera: 


\section{Tabla 1. Relación de respuestas con la inteligencia de negocios}

\begin{tabular}{|l|l|}
\hline \multicolumn{1}{|c|}{ Resultados de las entrevistas } & \multicolumn{1}{c|}{ Relación marco teórico } \\
\hline $\begin{array}{l}\text { Vinculación de empresas a programas de } \\
\text { apoyo }\end{array}$ & Sistemas para la integración vertical y horizontal \\
\hline Innovación & Todas las variables aplicadas \\
\hline Investigación de mercados & Big data y análisis de datos \\
\hline $\begin{array}{l}\text { Transferencia de conocimiento con otros } \\
\text { países }\end{array}$ & Sistemas para la integración vertical y horizontal \\
\hline La actualización permanente & Gestión de conocimiento \\
\hline $\begin{array}{l}\text { La gestión de conocimiento para dirigir las } \\
\text { empresas }\end{array}$ & $\begin{array}{l}\text { Sistemas de ejecución de fabricación software, } \\
\text { Integración de procesos y Sistemas de ejecución de } \\
\text { fabricación software }\end{array}$ \\
\hline Miedo al cambio & Hiperconectividad \\
\hline Cambio de tecnología & Robótica avanzada e inteligencia artificial \\
\hline
\end{tabular}

Fuente: Entrevistas realizadas en la 1IV Feria Mueble y Madera 2018

Las variables que más relevancia tienen, según los expertos entrevistados, son la base para mejorar los procesos y lograr la competitividad dentro del mercado; adicionalmente, la innovación es un punto clave para la empresa en donde la competencia es cada vez mayor y se requiere de un valor diferenciador para mejorar o desarrollar productos y servicios ofrecidos por la compañía.

Para conocer las opiniones de los representantes de las organizaciones, se realizaron 10 encuestas con las empresas que están en el parámetro de medianas o grandes, y que de acuerdo con la Ley 905 de 2004, "su patrimonio supera los 5.001 salarios mínimos mensuales legales vigentes": Primadera, Eurosierras Madecentro, Tablemac, Masisa, Julius Blum, Makita, Vauth Saguel, Kastamonu y Jowat. 
Tabla 2. Factores que inciden en la competitividad de las empresas, según representantes de grandes empresas

\begin{tabular}{|l|c|c|c|c|c|}
\hline \multicolumn{1}{|c|}{ Variable } & $\begin{array}{c}\text { Muy } \\
\text { importante }\end{array}$ & Importante & $\begin{array}{c}\text { Moderadamente } \\
\text { importante }\end{array}$ & $\begin{array}{c}\text { De poca } \\
\text { importancia }\end{array}$ & $\begin{array}{c}\text { Sin } \\
\text { importancia }\end{array}$ \\
\hline $\begin{array}{l}\text { Big data y análisis de } \\
\text { datos }\end{array}$ & 9 & 1 & 0 & 0 & 0 \\
\hline $\begin{array}{l}\text { Computación en la } \\
\text { nube }\end{array}$ & 7 & 1 & 1 & 1 & 0 \\
\hline Ciberseguridad & 8 & 2 & 0 & 0 & 0 \\
\hline Internet de las cosas & 7 & 3 & 0 & 0 & 0 \\
\hline $\begin{array}{l}\text { Integración de } \\
\text { procesos }\end{array}$ & 8 & 2 & 0 & 0 & 0 \\
\hline $\begin{array}{l}\text { Robótica avanzada e } \\
\text { inteligencia artificial }\end{array}$ & 6 & 3 & 1 & 0 & 0 \\
\hline $\begin{array}{l}\text { Sistemas para la } \\
\text { integración vertical y } \\
\text { horizontal }\end{array}$ & 7 & 3 & 0 & 0 & 0 \\
\hline Hiperconectividad & 9 & 0 & 1 & 0 & 0 \\
\hline $\begin{array}{l}\text { Fabricación digital } \\
\text { 3D) }\end{array}$ & 7 & 3 & 0 & 1 & 0 \\
\hline $\begin{array}{l}\text { Sistemas de ejecución } \\
\text { de fabricación software }\end{array}$ & 7 & 3 & 2 & & 0 \\
\hline $\begin{array}{l}\text { Realidad virtual y } \\
\text { aumentada }\end{array}$ & 6 & & & 0 & 0 \\
\hline
\end{tabular}

Fuente: Entrevistas realizadas en la 1IV Feria Mueble y Madera 2018

La percepción de las empresas más reconocidas del sector, las variables de hacen parte de la industria 4.0, están adquiriendo relevancia en la dirección organizacional, la calificación de cada variable se categoriza con más del 60\% entre muy importante e importante. También se realizaron 10 encuestas a visitantes de la feria del mueble y la madera, y que son propietarios o representantes de micro y pequeñas empresas "Su patrimonio es menor a los 5.000 salarios mínimos mensuales legales vigentes (Ley 905 de 2004)"se desarrolló el mismo ejercicio con el fin de conocer su percepción, la información se relaciona en la tabla 3.

Tabla 3. Factores que inciden en la competitividad de las empresas opinión representantes micro y pequeñas empresas

\begin{tabular}{|l|c|c|c|c|c|}
\hline \multicolumn{1}{|c|}{ Variable } & $\begin{array}{c}\text { Muy } \\
\text { importante }\end{array}$ & Importante & $\begin{array}{c}\text { Moderadamente } \\
\text { importante }\end{array}$ & $\begin{array}{c}\text { De poca } \\
\text { importancia }\end{array}$ & $\begin{array}{c}\text { Sin } \\
\text { importancia }\end{array}$ \\
\hline $\begin{array}{l}\text { Big data y análisis de } \\
\text { datos }\end{array}$ & 1 & 2 & 2 & 3 & 2 \\
\hline Computación en la nube & 2 & 2 & 2 & 3 & 1 \\
\hline Ciberseguridad & 1 & 3 & 3 & 3 & 0 \\
\hline Internet de las cosas & 2 & 3 & 3 & 2 & 0 \\
\hline Integración de procesos & 7 & 2 & 1 & 0 & 0 \\
\hline $\begin{array}{l}\text { Robótica avanzada e } \\
\text { inteligencia artificial }\end{array}$ & 1 & 3 & 5 & 1 & 0 \\
\hline
\end{tabular}




\begin{tabular}{|l|c|c|c|c|c|}
$\begin{array}{l}\text { Sistemas para la } \\
\text { integración vertical y } \\
\text { horizontal }\end{array}$ & 4 & 3 & 2 & 1 & 0 \\
\hline Hiperconectividad & 2 & 2 & 3 & 2 & 1 \\
\hline Fabricación digital (3D) & 1 & 1 & 2 & 4 & 2 \\
\hline $\begin{array}{l}\text { Sistemas de ejecución } \\
\text { de fabricación Software }\end{array}$ & 4 & 2 & 3 & 1 & 0 \\
\hline $\begin{array}{l}\text { Realidad virtual y } \\
\text { aumentada }\end{array}$ & 0 & 0 & 3 & 3 & 4 \\
\hline $\begin{array}{l}\text { Big data y análisis de } \\
\text { datos }\end{array}$ & 2 & 1 & 2 & 2 & 3 \\
\hline
\end{tabular}

Fuente: Entrevistas realizadas en la 1IV Feria Mueble y Madera 2018

Según las variables utilizadas, los representantes de micro o pequeñas empresas del sector del mueble y la madera consideraron que las variables de la industria 4.0 no son significativas para el desarrollo de la actividad económica, los factores que más importancia tiene son: la interacción de los procesos por medio de la internet de las cosas, sistemas para la integración vertical y horizontal, sistemas de ejecución de fabricación software; por su parte, los otros factores no tienen mayor incidencia en la gestión organizacional.

Al relacionar las respuestas de las grandes empresas sobre el uso de la inteligencia de negocios se identifica que, las entidades de mayor trayectoria y tamaño están desarrollando estrategias en donde incorporan sistemas de información inteligentes basados en la hiperconectividad e integración de procesos, además están en la búsqueda de tecnologías que mejoren sus procesos de producción y distribución. También resaltan la importancia de formar el talento humano para generar propuestas de valor en la organización.

Las micro o pequeñas empresas del sector del mueble y la madera, enfocan su gestión administrativa en la disminución de costos en las etapas de la cadena de suministros, no consideran prioridad la inteligencia de negocios en los procesos de la organización, las principales razones es el desconocimiento o evitar inversiones temporales que disminuyan su flujo de efectivo, el cual está comprometido para actividades que se generan en la operatividad de las organizaciones.
Para que las micro o pequeñas empresas conozcan la utilidad de la inteligencia de negocios en la industria del mueble, o tengan un apalancamiento financiero para la adquisición de tecnología, existen varias instituciones que promueven el fortalecimiento empresarial a través de líneas de crédito con tasas de interés blandas con periodos de gracias y formación, asesoría y acompañamiento en tecnología, información y comunicación.

\section{Entidades de promoción y apoyo}

Con el fin que el sector del mueble y la madera establezca una dinámica económica, y se genere desarrollo en todas las actividades comerciales que están involucradas en esta industria, a continuación, se relacionan las entidades públicas y privadas que tienen como objetivo la promoción del sector por medio de asesorías y financiamiento.

- La Cooperativa de la Industria del Mueble y la Madera: es una organización sin ánimo de lucro que invierte los aportes de los asociados en asesoría.

- La Federación Nacional de Industriales de la Madera (Fedemaderas): Representa a los productores de la industria de la madera, proveedores, distribuidores y comunidades dueñas de bosques naturales. Se encarga de promover acciones de pro del desarrollo del sector.

- La Asociación Colombiana de Fabricantes de Madera (Acemuebles): Promueve la educación de los agremiados por medio del Servicio Nacional 
de Aprendizaje (SENA).

- Asociación Colombiana de Ingenieros Forestales (ACIF): Busca el desarrollo del ejercicio profesional forestal, asesorando a los asociados en temas académicos, técnicos y científicos.

- Banco de Desarrollo Empresarial (Bancoldex): Promueve la competitividad a través de apalancamiento financiero o de asesoría empresarial.

- Procolombia: Asesora en actividades de comercio exterior, buscando el desarrollo en las empresas que quiera realizar inversión de capital extranjero, importaciones y exportaciones.

Con base en la opinión de especialistas, en la que manifestaron que la competitividad se está llevando a un nuevo escenario estratégico, en donde la tecnología se convierte en un instrumento necesario para todas las etapas de una cadena de suministro, se identificaron las organizaciones que pueden asesorar a las empresas del mueble y la madera en inteligencia de negocios, estas son:

- Tecnoparques: Es una organización del Estado y hace parte de los programas del SENA, esta institución asesora a empresas en cuatro líneas: Electrónica y telecomunicaciones, tecnologías virtuales, ingeniería y diseño y Biotecnología y nanotecnología.

- Tecnova: Gestiona el conocimiento en ciencias, tecnología e innovación articulando el conocimiento de las Instituciones de Educación Superior (IES), las empresas o sector real y el Estado.

- Parquesoft: Integra a las empresas por medio de eventos para intercambiar conocimiento al compartir experiencias de tecnología e innovación, es reconocida como un clúster de que integra a organizaciones de diferentes sectores.

- HugBog: Al igual que la anterior organización, su objetivo es integrar a las empresas por medio de eventos donde participan más de 200 organizaciones con el fin de impulsar proyectos innovadores.

- Opinno: Apoya a las empresas den modelos de gestión direccionados a la innovación y tec- nología, esta red internacional cuenta con publicaciones donde los participantes pueden participar.

- Apps.co: Promueve el uso de las tecnologías, información y comunicación, es una organización que está vinculada al Ministerio de las TIC y su objetivo se centra en el uso de páginas web y apps.

- Innpulsa: Encargada de promover el desarrollo buscando soluciones creativas a empresas que han presentado obstáculos en sus actividades.

- Connect Bogotá: Su objetivo es la articulación de las empresas con la gestión tecnológicas, su actividad se realiza con las organizaciones que están ubicadas en la ciudad de Bogotá.

- Centro de Innovación y Desarrollo Tecnológico (CIDT): Es una fundación sin ánimo de lucro que asesora a proyectos empresariales en innovación y tecnología.

- Colombia Digital: Ayuda a la formulación e implementación de estrategias de tecnología, información y comunicación. Está conformado por universidades, empresas e instituciones públicas.

- Corporación Ventures: Esta empresa gestiona fuentes de financiación para proyectos innovadores, sus programas de desarrollo se enfocan a la formación y aceleración de iniciativas empresariales. Entre los empresarios que se vinculan se realizan concursos para las empresas más creativas.

- Social Atom Ventures: Es una organización que brinda capital semilla, asesoría y acompañamiento a los proyectos lo necesiten. Se especializan en áreas de mercadeo, relaciones públicas e ingeniería de producto.

\section{Conclusiones}

Las nuevas tendencias de la sociedad 4.0 hace que la tecnología se convierta en un factor necesario para la planeación estratégica, la cual garantiza la sostenibilidad y el crecimiento. Las micro y pequeñas empresas del sector de muebles y madera desconocen la importancia de la industria 4.0 y su incidencia en la dinámica administrativa y comercial, aspecto que afecta el nivel de competitividad frente a las organizaciones que sí tienen en cuenta este factor. 
Los especialistas entrevistados en la investigación, corroboraron la poca importancia que tiene la inteligencia de negocios en las empresas que hacen parte de la industria del mueble y la madera, al manifestar que este sector enfrenta retos en materia de innovación, logística, comercialización, internacionalización, formalización y desarrollo empresarial. Aunque cuentan con una notable habilidad técnica, las micro y pequeñas empresas carecen de herramientas conceptuales que les permitan tomar decisiones asertivas enfocadas a una planeación estratégica y direccionada al cumplimiento de objetivos que generen un crecimiento sostenible y duradero.

Los representantes de las empresas que están en el parámetro de medianas o grandes empresas, entienden la inteligencia de negocios como una forma de responder a las exigencias del mercado de una manera oportuna, por eso hacen énfasis en direccionar parte de su presupuesto anual a la consecución de mejores equipos, maquinaria, software y capacitación de los colaboradores. Al revisar el desarrollo de la actividad económica de estas organizaciones, su plan estratégico está enfocado a la innovación en los productos, las estrategias de mercado, sus procesos internos, la gestión organizacional y consecución de mercados en el exterior.

Las pequeñas y micro empresas establecen un modelo de gerencia tradicional, donde la inteligencia de negocios tiene poca importancia, por lo anterior, la adquisición de herramientas tecnológicas no tiene respaldo en un presupuesto anual, que conllevaría a crear estrategias que modifiquen una gestión conservadora y proteccionista a una estructura organizacional donde las decisiones directivas se basen en variables e indicadores que faciliten diseñar procesos productivos acordes a la dinámica del mercado.

Para mejorar las prácticas empresariales que cambien la gestión en las organizaciones del sector, la inteligencia de negocios es una herramienta para ejecutar los procesos de manera rápida y eficiente con los recursos disponibles, con el fin de minimizar tiempos de producción y dar respuesta de manera oportuna a través de sistemas innovadores, que son adaptables a las necesidades de cada organización. Lo anterior, ayuda a que las empresas para que puedan posicionarse y conseguir una mayor participación en el mercado.

La inversión en las herramientas tecnológicas que promueven la industria 4.0 deben incluirse en el presupuesto anual de las organizaciones, con la condición de que se realice con base en un diagnóstico que sea pertinente a los recursos, tamaño y proyección que tenga cada empresa, lo anterior con el fin de no caer en inversiones inoficiosas que genere un uso negligente y que disminuya el capital de trabajo de las entidades del mueble y la madera.

Las entidades públicas y privadas que promueven el desarrollo de las industrias del mueble y la madera, deben crear un plan de comunicación con las pequeñas y medianas empresas para fomentar el uso de nuevas tecnologías con el apoyo de las organizaciones que asesoran, acompañan y financian, lo anterior con el fin de crear una red de conocimiento conjunto que fortalezca el sector.

Cuando las micro y pequeñas empresas decidan acoger la inteligencia de negocios en su plan estratégico, la academia, el sector privado y las instituciones públicas deben realizar investigaciones que caractericen las necesidades del mercado nacional e internacional, al mismo tiempo, promover la participación de los empresarios en eventos de innovación y desarrollo que mejoren la ingeniería del producto y de los procesos. 


\section{Referencias bibliográficas}

Abrego, D., Sánchez, T. y Medina, J. (2016). Influencia de los sistemas de información en los resultados organizacionales. Contaduría y Administración, 62, 303-320.

Aguirre, J. (2015). Inteligencia estratégica: un sistema para gestionar la innovación. Recuperado de https://www.icesi.edu.co/revistas/index.php/estudios_gerenciales/article/ view/1933/html_12

Allal-Chérif, O. y Bidan, M. (2016) Collaborative open training with serious games: Relations, culture, knowledge, innovation, and desire. Journal of Innovation \& Knowledge, 2 (1), 31-38

Ahumada, E. y Alberto, J. (2016). Inteligencia de negocios: estrategia para el desarrollo de competitividad en empresas de base tecnológica. Contaduría y Administración, 61, 127-158.

Barba, M. (2014). La habilidad de los directivos y su papel mediador entre formación e innovación. Revista Europea de Dirección y Economía de la Empresa, 23 (3), 127-136.

Bernal, C., Frost, J. y Sierra, H. (2015). Importancia de la gerencia del conocimiento: contrastes entre la teoría y la evidencia empírica. Estudios gerenciales, 30, 65-72.

Cano, J. y Baena, J. (2015). Tendencias en el uso de las tecnologías de información y comunicación para la negociación internacional. Estudios Gerenciales, 31 (136), 335-346.

Chang, V. (2016). Review and discussion: E-learning for academia and industry. International Journal of Information Management, 36 (3), 476-485
Chirinos, E., Rivero, E., Méndez, E., Goyo, A. y Figueredo, C. (2010). El Kaizen como un sistema actual de gestión personal para el éxito organizacional en la empresa ensambladora Toyota Negotium, 6 (16), 113-135.

DANE. (5 de abril de 2019). Encuesta anual manufacturera 2016. Recuperado de http:// www.dane.gov.co/index.php/estadisticaspor-tema/industria/encuesta-anual-manufacturera-enam

Díaz, D. (2 de noviembre de 2018). Diseño, clave para acelerar la industria de los muebles. Recuperado de https://www.eltiempo.com/ economia/sectores/industria-de-mueblesen-colombia-se-reinventa-con-tecnologiay-diseno-289074

Hernández, S., Fernández, C., y Baptista, P. (2014). Metodología de la investigación, sexta edición: México D.F.: Mc Graw Hill.

Ley 905 (Agosto 2 de 2004). Promoción del desarrollo de la micro, pequeña y mediana empresa colombiana. Recuperado de http:// www.mipymes.gov.co/temas-de-interes/ definicion-tamano-empresarial-micropequena-median

Leyva, M., Pérez, P., Febles, A. y Gulín, J. (2013). Mapas cognitivos difusos para la selección. Contaduría y Administración, 58, 95-117.

Luigi, S. (2015). The Plant Simulator as viable means to prevent and manage risk through competencies management: Experiment results. Safety Science, 84, 46-56.

Mendoza, J. y Valenzuela, A. (2014). Aprendizaje, innovación y gestión tecnológica en la pequeña empresa: Un estudio de las industrias metalmecánica y de tecnologías de información en Sonora. Contaduría y Administración, 59 (4), 253-284. 
Orta, E., Ruiz, M., Hurtado, N. y Gawn, D. (2014) Decision-making in IT service management: a simulation based approach. Decision Support Systems, 66, 36-51.

Revista Gerente (25 octubre 2018). Muebles de madera. Recuperado de http://gerente.com/ co/guias/muebles-madera-2/

Román, J. (10 de junio de 2019). Industria 4.0: la transformación digital de la industria. Facultad de Ingeniería de la Universidad de Deusto. Recuperado de http://coddii. org/wp-content/uploads/2016/10/InformeCODDII-Industria-4.0.pdf

Santoro, G. (2017). The Internet of Things: Building a knowledge management system for open innovation and knowledge management capacity. Technological Forecasting and Social Change, 136, 347-354.
Sivarajah, U., Mustafa, M., Irani, Z. y Weerakkod, V. (2016). Critical analysis of Big Data challenges and analytical methods. Journal of Business Research, 70, 263-286.

Tena-Chollet, F., Tixier, J., Dandrieux, A. y Slangen, P. (2016). Training decision-makers: Existing strategies for natural and technological crisis management and specifications of an improved simulation-based tool. Safety Science, 97, 144-153.

Vidal, D. y Domenge, R. (2017). Simulador de vuelo ejecutivo como medio de aprendizaje en la planeación de recursos de nuevas empresas bajo el enfoque del marcador balanceado. Contaduría y Administración, 62, 556-576. 